Agrotrópica 33(1): 39 - 48. 2021.

Centro de Pesquisas do Cacau, Ilhéus, Bahia, Brasil

\title{
OCORRÊNCIA E ASPECTOS BIOECOLÓGICOS DE Tetranychus urticae (ACARI: TETRANYCHIDAE) EM Acmella oleracea
}

\author{
Geraldo José Nascimento de Vasconcelos ${ }^{1 *}$, Fernanda Guimarães Paes ${ }^{2}$ \\ ${ }^{1}$ Universidade Federal do Amazonas, Instituto de Ciências Exatas e Tecnologia. Rua Nossa Senhora do Rosário, 3863 , \\ 69103-128, Tiradentes, Itacoatiara, AM, Brasil, gjnvasconcelos@yahoo.com.br. ${ }^{2}$ Agroecóloga, bolsista de Apoio Técnico \\ da Fundação de Amparo à Pesquisa do Estado do Amazonas, fgpaes@hotmail.com. \\ *Autor para correspondência: gjnvasconcelos@yahoo.com.br
}

\begin{abstract}
Um dos fatores que podem afetar a produção de jambu, Acmella oleracea (L.) R.K. Jansen, são os insetos e ácaros pragas. Entre as bases do manejo integrado de pragas estão a identificação e o conhecimento bioecológico. Assim, o estudo objetivou realizar o primeiro registro de ocorrência de Tetranychus urticae Koch, 1835 em jambu; caracterizar os danos causados; estabelecer o período do ano mais favorável para a ocorrência na região do estudo e; fornecer informações biológicas da praga no novo hospedeiro. Os registros de ocorrência foram realizados em setembro/2016 e agosto/2017 no município de Itacoatiara-AM. Os danos iniciaram com o surgimento de pequenas manchas, progredindo para grandes lesões cloróticas, em seguida, as bordas enrolaram e secaram. Para o local da ocorrência, os meses de temperatura mais elevada e menor umidade relativa do ar, agosto a outubro, provavelmente, são os mais favoráveis para a ocorrência de altas populações de T. urticae. No novo hospedeiro, o período de ovoadulto foi de 7,4 dias. A fecundidade foi de 67,9 ovos/fêmea, com taxa líquida de reprodução de 26,69 fêmea/fêmea/ geração. Assim, T. urticae está adaptado a cultura, podendo causar severos danos, sobretudo nos meses de menor umidade relativa do ar.
\end{abstract}

Palavras-chave: Agricultura tropical, jambu, ácaro-rajado, bioecologia de pragas.

Occurrence and bioecological aspects of Tetranychus urticae (Acari: Tetranychidae) in Acmella oleracea. One of the factors that can affect the production of Acmella oleracea (L.) R.K. Jansen are insects and pest mites. Among the steps of integrated pest management are identification and bioecological knowledge. Thus, the study aimed to make the first record of occurrence of Tetranychus urticae Koch, 1835 in A. oleracea; characterize the damage; establish the most favorable period of the year for the occurrence in the study region; and provide biological information of the pest on the new host. The occurrence records were performed in September/2016 and August/2017 in the municipality of Itacoatiara, State of Amazonas. The damage started with the appearance of small spots, progressing to large chlorotic lesions, after the border stay twisted and dried. For the place of occurrence, the months of higher temperature and lower relative humidity, August to October, are probably the most favorable for the occurrence of high populations of T. urticae. In the new host, the egg-adult period is 7.4 days. Females have fecundity of $67.9 \mathrm{eggs} / \mathrm{female}$. The a fecundity was $67.9 \mathrm{eggs} / \mathrm{female}$, with a net reproduction rate of $26.69 \mathrm{female} / \mathrm{female} /$ generation. Thus, T. urticae is adapted to A. oleracea, and can cause severe damage, especially in the months of lower relative humidity.

Key words: Tropical agriculture, jambu, two-spotted spider mite, pest bioecology. 


\section{Introdução}

O jambu, Acmella oleracea (L.) R.K. Jansen, também conhecido como abecedária, agrião-do-Pará, agrião-bravo, agrião-do-Brasil, agrião-do-Norte, botão-de-ouro, erva-maluca, jabuaçú, jambuaçú ou nhambu, é uma planta alimentícia não convencional da família Asteraceae (Favoreto e Gilbert, 2010; Homma et al., 2014). Apresenta porte herbácea com 20 a $40 \mathrm{~cm}$ de altura, ocorrendo em regiões tropicais e subtropicais da África, Ásia e América do Sul (Lewis et al., 1988; Favoreto e Gilbert, 2010).

Entre os fatores que podem afetar a produção do jambu estão os insetos e ácaros pragas. Insetos como paquinha, grilo, lagarta-rosca, pulgão e besouros desfolhadores são citados como praga em áreas de cultivo (Cardoso e Garcia, 1997). Entre os ácaros fitófagos há registros para duas espécies em jambu, o ácaro branco (Polyphagotarsonemus latus (Banks, 1904)) da família Tarsonemidae (Vasconcelos, Lima e Silva, 2012) e o ácaro de teia (Tetranychus neocaledonicus André, 1933) pertencente à família Tetranychidae (Migeon, 2015).

Mundialmente, diversas espécies de ácaros são consideradas como pragas agrícolas. Entre essas, os ácaros de teia ou tetraniquídeos são as principais, com cerca de 100 espécies de importância econômica (Hoy, 2011). A mais conhecida dessas espécies é o Tetranychus urticae Koch, 1835 (Moraes e Flechtmann, 2008; Hoy, 2011), a qual ocorre em 124 países, distribuídos nas seis regiões zoogeográficas, com registro em 1.169 plantas hospedeiras (Migeon \& Dorkeld, 2019).

Tetranychus urticae é altamente variável em sua biologia, comportamento e ecologia (Hoy, 2011). O desenvolvimento e reprodução, podem diferir de acordo com a ampla gama de plantas hospedeiras e condições ambientais das diferentes regiões de ocorrência. Outra variação importante está no padrão de cor que a espécie pode apresentar, podendo ser rajado (idiossoma verde com manchas escuras nas laterais) ou vermelho. O padrão vermelho já foi considerado como Tetranychus cinnabarinus Boisduval, 1867. No entanto, estudos morfológicos, biológicos e moleculares confirmam que se trata de uma sinonímia de T. urticae (Auger et al., 2013).
Entre as bases do manejo integrado de pragas em agroecossistemas estão a identificação da praga e o conhecimento bioecológico dessa (Stein, 2006; EhiEromosele, Nwinyi \& Ajani, 2013). A identificação é o primeiro passo para a solução de um problema biológico, a exemplo de desequilíbrios populacionais, sendo fundamental para possibilitar a catalogação de todas as informações já documentadas sobre a praga (Zucchi, 1990). Por sua vez, o conhecimento da bioecológico da praga é fundamental no estabelecimento das estratégias de manejo (EhiEromosele, Nwinyi \& Ajani, 2013).

Tendo em vista a importância da identificação, tanto da praga quanto dos seus danos, e do conhecimento da bioecologia da mesma, os objetivos do estudo foram relatar pela primeira vez a ocorrência de T. urticae em jambu; caracterizar os danos causados; estabelecer o período do ano em que esse ácaro pode atingir maior população na região de ocorrência e fornecer informações de desenvolvimento nesse novo hospedeiro.

\section{Material e Métodos}

Os registros de ocorrência foram realizados no município de Itacoatiara, Estado do Amazonas. Os estudos de biologia foram realizados no Laboratório do Instituto de Ciências Exatas e Tecnologia (ICET) da Universidade Federal do Amazonas (UFAM).

Caracterização das áreas de ocorrência de $T$. urticae em jambu.

As ocorrências foram registradas em áreas de produção diversificada de olerícolas, principalmente, folhosas e plantas medicinais de consumo local, que, além do jambu, são representadas, sobretudo, por basílico (Ocimum basilicum L.), cariru (Talinum triangulare (Jacq.) Willd.), cebolinha (Allium fistulosum L. e A. schoenoprasum L.), chicória (Cichorium endivia L.), coentro (Coriandrum sativum L.), couve (Brassica oleracea L. var. acephala DC.), mastruz (Chenopodium ambrosioides L.) e hortelã (Mentha $x$ villosa Huds.). Essas áreas são localmente caracterizadas pela produção agrícola familiar, em pequena escala, nas regiões periféricas das áreas urbanas. Nessas áreas, as olerícolas são cultivadas em canteiros, no solo ou suspensos. 
Conforme os agricultores, os canteiros foram preparados com uma mistura de solo de várzea e "paú" (denominação local para alguns tipos de madeira em decomposição) de mungubeira (Pseudobombax munguba (Mart. e Zucc.) Dugand) e/ou inajazeiro (Maximiliana maripa (Aubl.) Drude). A quantidade de "paú" por canteiro variou, entre 20 a $50 \mathrm{~L} / \mathrm{m}^{2}$, conforme a disponibilidade.

O jambu foi propagado nos canteiros através da técnica de estaquia, com espaçamento de $5 \mathrm{~cm}$ entre estacas e $10 \mathrm{~cm}$ entre linhas. Os tratos culturais adotados pelos agricultores foram capinas manuais $\mathrm{e}$ rega diária das plantas, em dias sem chuva, disponibilizando, através de um regador, aproximadamente $5 \mathrm{~L}$ de água $/ \mathrm{m}^{2}$ de canteiro.

\section{Ocorrência e caracterização dos danos de $T$. urticae em jambu.}

A ocorrência de T. urticae em jambu foi registrada em 24/09/2016 (3 08' 10" S; 58 26' 19" O e altitude $23 \mathrm{~m}$ ) e 01/08/2017 ( $3^{\circ} 07^{\prime} 41^{\prime \prime} \mathrm{S} ; 58^{\circ} 26^{\prime} 35^{\prime \prime} \mathrm{O}$ e altitude $19 \mathrm{~m}$ ), em plantas propagadas a cerca de $60 \mathrm{e}$ 35 dias antes da ocorrência, respectivamente. Após as ocorrências os canteiros continuaram sendo observados por 15 dias para caracterizar os danos e a evolução da população do ácaro na planta.

Amostras de ramos infestados foram coletadas e levadas ao laboratório, onde machos e fêmeas adultas foram montados, com auxílio de estereomicroscópio, em lâmina para microscopia com meio de Hoyer (Moraes e Flechtmann, 2008). As lâminas foram levadas à estufa para secagem, a $45^{\circ} \mathrm{C}$ por 3 dias. Em seguida os ácaros foram identificados, em microscópio com contraste de fases (OPTICAM O400 Standard), com base nas características morfológicas descritas por Pritchard \& Baker (1955) e Jeppson, Keifer \& Baker (1975).

As médias para a temperatura e umidade relativa do ar e o acumulado da precipitação na área urbana de Itacoatiara, nos 30 dias que precederam as coletas foram obtidos no site do Instituto Nacional de Meteorologia (INMET, 2020), com os dados aferidos na estação meteorológica do município $\left(03^{\circ} 08^{\prime} 14^{\prime \prime} \mathrm{S}\right.$; $58^{\circ} 26^{\prime} 34^{\prime \prime} \mathrm{O}$ e altitude $19 \mathrm{~m}$ ).

Condições climáticas que podem favorecer a ocorrência de T. urticae em jambu ao longo do ano.

Para o estabelecimento das condições climáticas de temperatura, umidade relativa do ar e precipitação mais favoráveis para T. urticae ao longo do ano em Itacoatiara, foram utilizados dados mensais da década de 01/01/2010 a 31/12/2019, obtidos no site do INMET (2020), com os dados aferidos na estação meteorológica do município. Em seguida, as condições climáticas mensais foram confrontadas com os parâmetros ideais, já estabelecido na literatura, para T. urticae, com base em Hazan, Gerson \& Tahori (1973), Klubertanz, Pedigo \& Carlson (1990) Kim et al. (2008), Abd El-Wahed \& El-Halawany (2012), Riahi et al. (2013), Ali et al. (2017), Maula \& Khan (2016) e Kaur \& Zalom (2018).

Desenvolvimento, reprodução, tabela de vida de fertilidade e curva de sobrevivência de $T$. urticae em jambu.

Os estudos foram realizados com T. urticae da criação estoque, a qual foi iniciada a partir de indivíduos coletados nas plantas de jambu na ocorrência de 2016, e mantidos em laboratório, em ramos com folhas da mesma planta. Tanto a criação quanto os estudos foram conduzidos em laboratório, sob as condições de $27,8 \pm 2,78^{\circ} \mathrm{C}, 76,7 \pm 16,21 \%$ de UR e fotoperíodo de aproximadamente $12 \mathrm{~h}$.

Inicialmente, 60 fêmeas adultas de T. urticae foram retiradas da criação e colocadas em uma unidade experimental, semelhante à descrita por Silva et al. (2009). Em seguida, a folha de jambu, utilizada na confecção da unidade, foi inspecionada para remoção de possíveis artrópodes. As fêmeas permaneceram ovipositando na unidade experimental por $12 \mathrm{~h}$. Os ovos obtidos foram observados a cada $12 \mathrm{~h}$ para determinação do período de incubação e da viabilidade.

Após a eclosão, as larvas foram individualizadas em unidades experimentais. As observações continuaram a cada $12 \mathrm{~h}$ para determinação da duração e viabilidade das fases de larva e, posteriormente, de protoninfa e deutoninfa. Após a emergência, os adultos foram sexados e as fêmeas foram mantidas permanentemente acasaladas. Os adultos foram avaliados diariamente, observando o número de ovos e a sobrevivência. Durante todo o estudo com as fases pós-embrionárias, a cada três dias os ácaros foram transferidos para uma nova unidade.

\section{Análise dos dados.}

No estudo de condições climáticas favoráveis a 
ocorrência de T. urticae na região, os meses com as condições semelhantes foram agrupados. Para esse fim, as médias mensais de cada parâmetro foram submetidas a teste de normalidade e homogeneidade da variância e, quando necessário foram transformadas em $(x+0,5)^{1 / 2}$. Em seguida, as médias foram submetidas à Análise de Variância e agrupadas pelo teste de ScottKnott (Scott \& Knott, 1974).

Para os estudos biológicos, as médias de duração das fases de ovo, larva, protoninfa e deutoninfa, para a população e individualmente para cada sexo, além da sobrevivência entre as fases e a proporção de machos e fêmeas na população, foram comparadas através do $\mathrm{IC}_{95 \%}$ para a diferença entre duas médias $\left(\mathrm{IC}_{\text {DEDM }}\right.$ ) (Miao \& Chiou, 2008; Tan \& Tan, 2010). Na fase adulta, o $\mathrm{IC}_{\text {DEDM }}$ também foi utilizado para comparar os períodos de pré-oviposição, oviposição e pós-oviposição, além da longevidade entre machos e fêmeas.

A tabela de vida de fertilidade foi elaborada conforme Southwood \& Henderson (2000). Posteriormente, os parâmetros da tabela de vida, bem como a sobrevivência e a razão sexual, foram submetidos ao método Jackknife (Maia, Luiz \& Campanhola, 2000) e tiveram as médias e respectivos erros padrões estabelecidos.

A distribuição de Weibull foi utilizada para descrever a sobrevivência da população (Reis e Haddad, 1997; Bouras \& Papadoulis, 2005). Em seguida, os valores dos parâmetros de forma $(c)$ e de escala $(b)$ foram utilizados para estabelecer o ciclo média de vida da população (Reis e Haddad, 1997). O parâmetro $C$ também foi usado para classificar a curva de sobrevivência, como do tipo I, II ou III (Pinder, Wiener \& Smith, 1978). Para descrever o grau de concordância entre a sobrevivência observada nas avaliações e a sobrevivência estimada pela distribuição de Weibull, foi determinado o coeficiente de regressão não linear $\left(\mathrm{R}^{2}\right)$ (Olkin \& Finn, 1995).

Todas às análises foram realizadas com auxílio do Software R 3.5.1 (R Core Team, 2018), em nível de $5 \%$ de significância $(p \leq 0,05)$.

\section{Resultados e Discussões}

Ocorrência e caracterização dos danos de $T$. urticae em jambu.

Este é o primeiro relato de T. urticae em jambu.
As populações registradas nas duas ocorrências apresentaram o padrão de coloração vermelho (Figuras 1A e B). Inicialmente as colônias foram encontradas na superfície abaxial das folhas mais velhas (medianas e basais). Com o aumento da população, cerca de 5 dias após a constatação da ocorrência, os ácaros também foram encontrados na superfície adaxial e em folhas mais novas (Figuras 1A e D). A quantidade de teia também aumentou consideravelmente, cerca de 10 dias após a constatação da ocorrência, recobrindo praticamente toda a planta (Figura 1E).

Os danos observados são os típicos para ácaros tetraniquídeos, assim, inicialmente as folhas apresentaram pequenas manchas verde-pálidas (Figura 1B), que com o tempo se tornam grandes lesões cloróticas (Figuras 1D e E), as bordas das folhas podem enrolar e secar (Figuras 1C e F). Por fim, as folhas mais velhas murcharam e secaram totalmente (Figura F).

Os danos causados, tanto na ocorrência de 2016 quanto de 2017, levaram as plantas a morte. Nesses anos a média da temperatura e umidade relativa do ar e o acumulado da precipitação, para os 30 dias que precederam o registro, foram de $28,7{ }^{\circ} \mathrm{C}, 75,1 \%$ e $79,3 \mathrm{~mm}$ e $28,1^{\circ} \mathrm{C}$, $75,4 \%$ e $181,1 \mathrm{~mm}$, respectivamente para cada parâmetro climático e ano de coleta (INMET, 2020).

Condições climáticas que podem favorecer a ocorrência de T. urticae em jambu ao longo do ano.

Nas condições climáticas anuais do município de Itacoatiara, os meses mais quentes $(\mathrm{GL}=11 ; 108, \mathrm{~F}=$ 17,91 e $p<2,2.10^{-16}$ ) são os de agosto a novembro (Tabela 1). O oposto é observado para os meses de janeiro a abril. Para a umidade relativa do ar, os meses com as maiores umidades $(\mathrm{GL}=11 ; 108, \mathrm{~F}=25,47 \mathrm{e}$ $p<2,2.10^{-16}$ ) foram de janeiro a maio, já as menores umidades foram observadas para os meses de agosto a outubro. O acumulado da precipitação mensal demonstra que os meses de fevereiro a abril são os de maiores precipitações $(\mathrm{GL}=11 ; 108, \mathrm{~F}=21,61 \mathrm{e}$ $\left.p<2,2.10^{-16}\right)$, enquanto agosto e setembro apresentaram as menores precipitações.

Para T. urticae, a duração e a sobrevivência das fases imaturas, bem como a fecundidade das fêmeas são favorecidas entre as temperaturas de 24 a $32{ }^{\circ} \mathrm{C}$. Nessa condição o tempo de desenvolvimento é mais curto, a sobrevivência é maior e a fecundidade é mais 

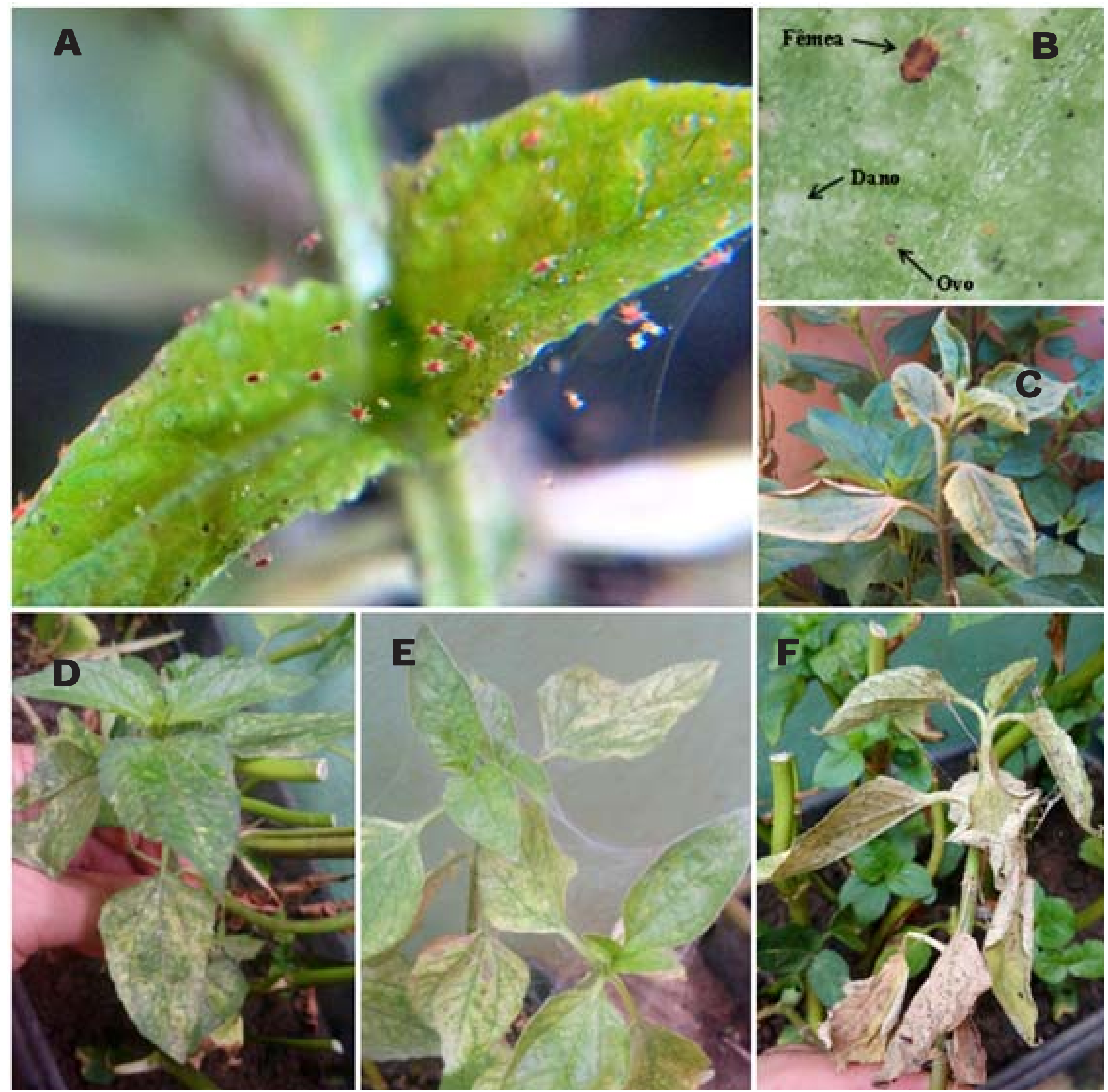

Figura 1. Tetranychus urticae (padrão vermelho) em folha de jambu. A - Colônia e teia em folhas apicais. B - Colônia e danos na face abaxial, vista com auxílio de estereomicroscópio. C - Secamento e enrolamento das bordas das folhas medianas. D e E - Folhas medianas e apical cloróticas e recobertas por teia, 5 e 10 dias após a constatação da ocorrência, respectivamente. F - Folhas medianas murchas e basais totalmente secas.

elevada, principalmente nas temperaturas mais altas desse intervalo (Hazan, Gerson \& Tahori, 1973; Kim et al., 2008; Abd El-Wahed \& El-halawany, 2012; Riahi et al., 2013; Ali et al., 2017; Maula \& Khan, 2016; Kaur \& Zalom, 2018). Dessa forma, as oscilações das temperaturas mensais em Itacoatiara, 26,8 a $29,0{ }^{\circ} \mathrm{C}$, ficam sempre dentro da faixa de temperatura favorável para o desenvolvimento de T. urticae. Na faixa de temperatura favorável, esse ácaro pode ter redução de 1,6 dias na duração do período de ovo-adulto, a cada um grau de incremento na temperatura (Kim et al., 2008).

A faixa de umidade relativa do ar mais apropriada a T. urticae é entre 22 e $80 \%$, favorecendo-o tanto na 
Tabela 1. Média mensal ( \pm Desvio Padrão) da temperatura, umidade relativa do ar e do acumulado da precipitação para o período de $01 / 01 / 2010$ a 31/12/2019 em Itacoatiara-AM

\begin{tabular}{|c|c|c|c|c|c|}
\hline \multirow{2}{*}{$\begin{array}{c}\text { Mês }^{1} \\
\text { Janeiro }\end{array}$} & \multicolumn{2}{|c|}{$\begin{array}{c}\text { Temperatura } \\
\left({ }^{\circ} \mathrm{C}\right)^{2} \\
\end{array}$} & $\begin{array}{c}\text { Umidade Relativa } \\
\text { do } \operatorname{Ar}(\%)^{2}\end{array}$ & \multicolumn{2}{|l|}{$\begin{array}{l}\text { Precipitação } \\
(\mathrm{mm})^{2 \mathrm{e} 3}\end{array}$} \\
\hline & $27,1 \pm 0,75$ & $\mathrm{C}$ & $83,8 \pm 3,17 \quad \mathrm{~A}$ & $281,2 \pm 94,23$ & B \\
\hline Fevereiro & $26,8 \pm 0,60$ & $\mathrm{C}$ & $84,4 \pm 2,01 \quad \mathrm{~A}$ & $347,2 \pm 100,64$ & A \\
\hline Março & $27,2 \pm 0,61$ & $\mathrm{C}$ & $83,5 \pm 3,25 \quad \mathrm{~A}$ & $449,7 \pm 166,81$ & A \\
\hline Abril & $27,1 \pm 0,35$ & $\mathrm{C}$ & $84,8 \pm 1,97 \quad \mathrm{~A}$ & $382,4 \pm 73,20$ & A \\
\hline Maio & $27,5 \pm 0,50$ & B & $83,9 \pm 1,92 \quad \mathrm{~A}$ & $293,9 \pm 120,93$ & B \\
\hline Junho & $27,9 \pm 0,32$ & B & $80,0 \pm 1,49$ В & $166,7 \pm 64,99$ & $\mathrm{C}$ \\
\hline Julho & $27,9 \pm 0,43$ & B & $78,7 \pm 2,86 \mathrm{C}$ & $124,3 \pm 44,81$ & $\mathrm{C}$ \\
\hline Agosto & $28,7 \pm 0,43$ & A & $74,5 \pm 3,32 \mathrm{D}$ & $67,3 \pm 43,99$ & $\mathrm{D}$ \\
\hline Setembro & $29,0 \pm 0,40$ & A & $74,1 \pm 2,61 \quad \mathrm{D}$ & $57,3 \pm 33,48$ & $\mathrm{D}$ \\
\hline Outubro & $28,9 \pm 0,68$ & A & $74,8 \pm 3,35 \quad \mathrm{D}$ & $140,3 \pm 90,67$ & $\mathrm{C}$ \\
\hline Novembro & $28,4 \pm 0,62$ & A & $78,3 \pm 1,87 \quad \mathrm{C}$ & $138,2 \pm 67,86$ & $\mathrm{C}$ \\
\hline Dezembro & $27,5 \pm 0,82$ & B & $82,2 \pm 1,97 \quad$ B & $291,1 \pm 125,66$ & B \\
\hline $\begin{array}{l}\text { Normalidade } \\
\left(p_{\text {Kolmogorov-Smirno }}\right.\end{array}$ & ) & & 0,10 & 0,78 & \\
\hline$\underset{\left(p_{\text {Bartlett }}\right)}{\text { Homogeneidad }}$ & 0,12 & & 0,23 & 0,25 & \\
\hline $\begin{array}{c}\text { Coeficiente de } \\
\text { Variação (\%) }\end{array}$ & 2,03 & & 3,20 & 21,78 & \\
\hline
\end{tabular}

${ }^{1}$ Dados disponibilizados pelo INMET (2020).

${ }^{2}$ Médias, seguidas pela mesma letra, na coluna, formam um grupo homogêneo de acordo com o teste de Scott-Knott ao nível de 5\% de probabilidade. ${ }^{3}$ Dados transformados em $(x+0,5)^{1 / 2}$.

fase jovem quanto na adulta, podendo propiciar menor mortalidade, principalmente dos jovens, e maior fecundidade (Hazan, Gerson \& Tahori, 1973). Condições de umidade a partir de $78 \%$, geralmente, tendem a suprimir grandes danos causados pelos ácaros, levando a redução da população (Boudreaux, 1958; Pal et al., 1989). Assim, a umidade relativa do ar em Itacoatiara pode ser um fator adverso determinante para a população de T. urticae, durante alguns meses do ano, sobretudo de janeiro a maio, que apresentam média de umidade superior a $80 \%$. Além disso, altas umidades também podem favorecer a ocorrência de doenças na população do ácaro, principalmente as causadas por fungos (Hazan, Gerson \& Tahori, 1973). Já os meses de agosto a outubro, provavelmente, são os mais favoráveis para a ocorrência de $T$. urticae em altas populações em Itacoatiara, por serem os meses de menores umidades, além de fazerem parte do agrupamento dos meses de maiores temperaturas.

A respeito da precipitação, Klubertanz, Pedigo \& Carlson (1990) verificaram que não há influência direta dessa sobre a população de T. urticae. A ocorrência preferencial do ácaro na superfície abaxial da folha, provavelmente, minimiza o efeito mecânico de remoção pela chuva, fazendo com que, esse fator, não influencie na abundância da população (Abreu et al., 2014).

Durante os meses com umidade mais favoráveis, a temperatura média foi de 28,9 ${ }^{\circ} \mathrm{C}$. Levando em consideração que a constante térmica $(K)$ e a temperatura base inferior de desenvolvimento $(\mathrm{Tb})$ para T. urticae são de 136,43 graus-dias e 13,79 ${ }^{\circ} \mathrm{C}$, respectivamente (Riahi et al., 2013), é possível estimar o número de gerações que podem ocorrer em campo nesses meses, através da equação proposta por Réaumur (1735). Assim, estima-se, para a localidade do estudo, que nos meses mais favoráveis possam ocorrer 10 gerações de T. urticae em campo, com rápido desenvolvimento, maior sobrevivência e alta fecundidade, possibilitando o surto populacional da praga.

Desenvolvimento, reprodução, tabela de vida de fertilidade e curva de sobrevivência de $T$. urticae em jambu.

Entre as fases de desenvolvimento de T. urticae em jambu, a mais longa foi a de ovo ( $\mathrm{IC}_{\mathrm{DEDM}}$ inferior $\geq 1,18$ ) (Tabela 2). O período de ovo-adulto durou 7,4 dias. Para machos e fêmeas, a duração de cada fase de desenvolvimento e do período de ovo-adulto, não diferiram $\left(\mathrm{IC}_{\mathrm{DEDM}}\right.$ inferior $\leq-0,04$ e superior $\geq$ $0,25)$.

A sobrevivência foi superior a $90 \%$ em todas as fases de desenvolvimento (Tabela 2), não havendo diferença nesse parâmetro entre as fases ( $\mathrm{IC}_{\mathrm{DEDM}}$ inferior $\leq-1,50$ e superior $\geq 6,64)$. Foi observada uma maior proporção de fêmeas na população ( $\mathrm{IC}_{\mathrm{DEDM}}$ inferior $=18,08$ ), a qual apresentou razão sexual de $67,9 \%$.

Uddin et al. (2015), Golizadeh et al. (2017) e Kumral et al. (2019) estudaram a biologia de T. urticae em diferentes hospedeiros, com temperaturas e umidades relativas do ar variando de 24 a $32{ }^{\circ} \mathrm{C}$ e de 50 a $70 \%$, respectivamente. Nesses estudos, o desenvolvimento variou de 3,1 a 4,9, 0,9 a 2,6, 1,6 a 
Tabela 2. Média ( \pm Erro Padrão) da duração das fases de ovo, larva, protoninfa e deutoninfa, período de ovo-adulto, sobrevivência, longevidade, pré-oviposição, oviposição, pós-oviposição, fecundidade total e diária e razão sexual para uma população de Tetranychus urticae em folhas de jambu (Acmella oleracea)

\begin{tabular}{|c|c|c|c|c|}
\hline Imaturo $^{1}$ & 우 $(\mathrm{n}=36)$ & $\widehat{\partial}(\mathrm{n}=17)$ & 주우 $(\mathrm{n}=56) \mathrm{S}$ & $\begin{array}{l}\text { Sobrevivência } \\
(\%)^{3}\end{array}$ \\
\hline 080 (aras) & $3,0 \pm 0,00 \mathrm{aA}^{2}$ & $3,1 \pm 0$ & $3,0 \pm 0,01 \mathrm{a}$ & ба \\
\hline va (dias) & 1,4 & 1,4 & & $94 a$ \\
\hline ias) & 1,5 & & & \\
\hline & 1,6 & & & $05 \mathrm{a}$ \\
\hline Ovo-adulto (dias) & $7,5 \pm$ & $7,2=$ & & $84,1 \pm 0,03$ \\
\hline \multicolumn{2}{|l|}{ Adulto $^{1}$} & 우 $(\mathrm{n}=32)$ & $\sigma^{\lambda}(\mathrm{n}=12)$ & 주우 $(n=44)$ \\
\hline \multicolumn{2}{|l|}{ Longevidade (dias) } & $14,7 \pm 1,40 \mathrm{~A}^{2}$ & $6,8 \pm 1,00 \mathrm{~B}$ & $12,5 \pm 1,18$ \\
\hline \multicolumn{2}{|c|}{ Pré-oviposição (dias) } & & - & - \\
\hline \multicolumn{2}{|l|}{ Oviposição (dias) } & & - & - \\
\hline \multicolumn{2}{|c|}{ Pós-oviposição (dias) } & & - & - \\
\hline \multicolumn{2}{|c|}{ Fecundidade total (ovos/fêmea) } & 67 & - & - \\
\hline \multicolumn{2}{|c|}{ Fecundidade diária (ovos/fềmea/dia) } & $4,7 \pm 0,36$ & - & - \\
\hline \multicolumn{2}{|l|}{ Razão sexual $(\%)^{3}$} & - & - & $67,9 \pm 0,12$ \\
\hline \multicolumn{5}{|c|}{$\begin{array}{l}\text { e fotoperíodo de aproximadamente } 12 \mathrm{~h} \text {. } \\
{ }^{2} \text { Médias, para imaturos ou adultos, seguidas da mesma letra, minúscula na } \\
\text { coluna ou maiúscula na linha, não diferem entre si pelo } \mathrm{IC}_{95 \%} \text { para a diferença } \\
\text { entre duas médias }\left(\mathrm{IC}_{\mathrm{DEDM}}\right) \text {. }{ }^{3} \text { Erro padrão determinado a partir de pseudoreplicatas } \\
\text { pelo método Jackknife. }\end{array}$} \\
\hline
\end{tabular}

2,2, 1,6 a 2,6, 9,2 a 12,3 dias, respectivamente para ovo, larva, protoninfa, deutoninfa e período de ovo-adulto, com sobrevivência variando de 56 a $96 \%$ e razão sexual entre 66 a 85\%. Assim, a duração das fases imaturas, a sobrevivência e a razão sexual, observadas no presente estudo, estão de acordo com os intervalos observados nos estudos citados. Já o período de ovo-adulto foi inferior aos observados naqueles estudos.

As fêmeas apresentaram longevidade de 14,7 dias (Tabela 2), com o período de oviposição sendo o mais longo durante a longevidade, com 12,7 dias $\left(\mathrm{IC}_{\mathrm{DEDM}}\right.$ inferior $\geq 10,02$ ). Ao longo desse período, a fecundidade foi de 67,9 ovos/fêmea. Para os machos, a longevidade foi de 6,8 dias, sendo menos longevos que as fêmeas $\left(\mathrm{IC}_{\text {DEDM }}\right.$ inferior $\left.=6,39\right)$.

Os valores da longevidade e fecundidade das fêmeas da população de T. urticae, também estão de acordo com os intervalos registrados para esse ácaro em outros hospedeiros, onde a longevidade variou de 13,2 a 28,9 dias e a fecundidade de 45,5 a 125,2 ovos/fêmea (Uddin et al., 2015, Golizadeh et al., 2017 e Kumral et al., 2019).

Com base nos parâmetros da tabela de vida de fertilidade, obtidos para a população de T. urticae
(Tabela 3), observa-se que o tempo médio de cada geração $(T)$ e o tempo para duplicação da população (TD) são de 16,1 e 2,8 dias, respectivamente. Essa população pode ter a capacidade de aumento $\left(R_{0}\right)$ de 26,7 fêmea/fêmea/ geração, apresentando taxa intrínseca de crescimento corrigida $\left(r_{m}{ }^{\prime}\right)$ e taxa finita de aumento $(\lambda)$ de 0,244 e 1,276 progênie/ fêmea/dia, respectivamente.

Nos estudos realizados por Uddin et al. (2015), Golizadeh et al. (2017) e Kumral et al. (2019), os parâmetros das tabelas de vida de $T$. urticae, em diferentes hospedeiros e condições ambientais, foram 13,3 a 21,2 dias, 2,6 a 4,1 dias, 26,7 a 82,4 fêmea/fêmea/geração, 0,169 a 0,269 progênie/fêmea/dia e 1,184 a 1,307 progênie/fêmea/dia, respectivamente para $T, T D, R_{0}, r_{m}$ ' e $\lambda$. Dessa forma, os valores obtidos para os parâmetros da tabela de vida, da população de T. urticae em jambu, também estão de acordo com os estudos citados e demonstram que há crescimento populacional, uma vez que o $r_{m}$ ' foi maior que zero e o $\lambda$ maior que um (Southwood $\&$ Henderson, 2000).

A fertilidade específica $(m x)$ mostra uma flutuação irregular ao longo do período de oviposição (Figura 2). Ao longo do ciclo de vida, no décimo quinto e vigésimo quinto dia, após a eclosão, ocorreram os principais picos de oviposição.

A $m x$ também confirma a boa adaptação de $T$. urticae ao jambu, apresentando um padrão semelhante ao observado em outros hospedeiros onde essa espécie é praga, como diferentes variedades de Rosa spp. e cultivares de Solanum melongena L. (Golizadeh et al., 2017; Kumral et al., 2019).

A distribuição de frequência de Weibull foi um modelo apropriado para descrever a sobrevivência em função da idade das fêmeas adultas ( $\mathrm{GL}=37, \mathrm{c}^{2}=$ $27,01, p=0,89$ ), tendo o $\mathrm{R}^{2}$ de $95,1 \%$ (Figura 2). Os parâmetros $c$ e $b$ foram 2,11 e 21,69, respectivamente, sendo ambos significativamente maior que zero $(p<$ $\left.2,2 \cdot 10^{-16}\right)$. Com base nesses parâmetros, o ciclo médio de vida da população de T. urticae em jambu é de 19,2 dias, apresentando uma curva de sobrevivência do tipo I $(c>1)$. 
Tabela 3. Estimativa dos parâmetros da tabela de vida de fertilidade para uma população de Tetranychus urticae em folhas de jambu (Acmella oleracea)

\begin{tabular}{|c|c|}
\hline Parâmetro $^{1}$ & Médias \pm Erro Padrão ${ }^{2}(n=32)$ \\
\hline Tempo entre cada geração $(T)$ & $16,1 \pm 0,021$ dias \\
\hline Tempo para duplicação da população (TD) & $2,8 \pm 0,003$ dias \\
\hline Taxa líquida de reprodução $\left(R_{0}\right)$ & $26,7 \pm 0,123$ ㅇ / ㅇ /geração \\
\hline Capacidade inata de aumento em número corrigida $\left(r_{m}{ }^{\prime}\right)$ & $0,244 \pm 0,0002$ progênie/ 우 /dia \\
\hline Razão finita de aumento $(\lambda)$ & $1,276 \pm 0,0003$ progênie/ + + dia \\
\hline
\end{tabular}

${ }^{1}$ Condições ambientais no laboratório: $\mathrm{T}=27,8 \pm 2,78^{\circ} \mathrm{C}, \mathrm{UR}=76,7 \pm 16,21 \% \mathrm{e}$ fotoperíodo de aproximadamente $12 \mathrm{~h}$.

${ }^{2}$ Erro padrão determinado a partir de pseudoreplicatas pelo método Jackknife.

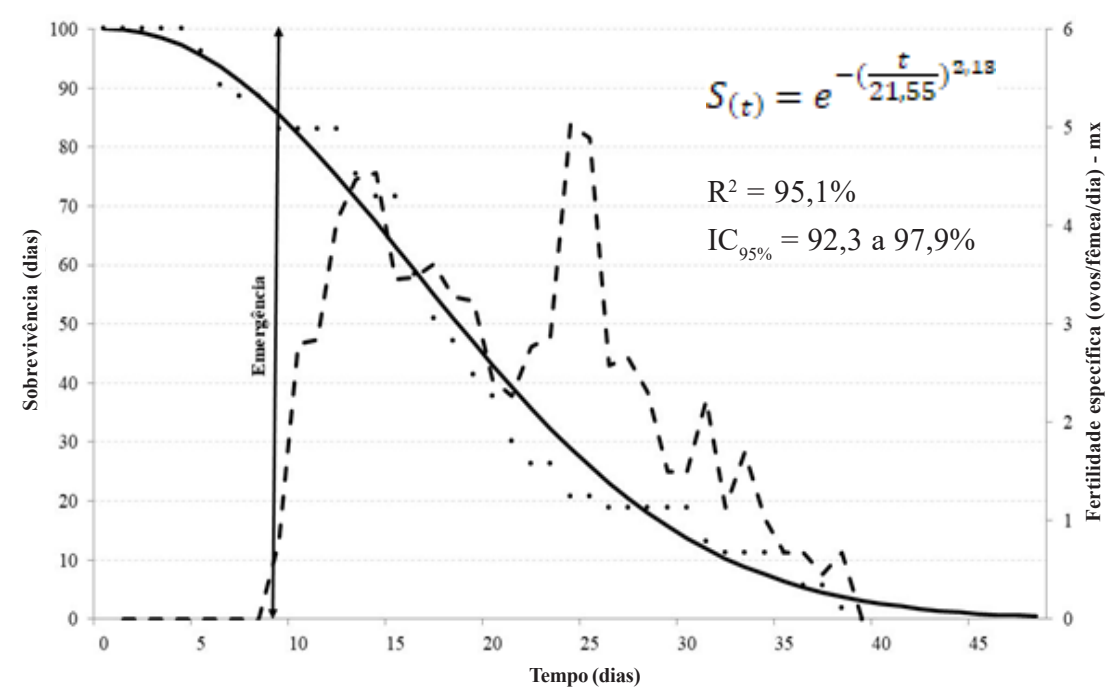

Figura 2. Fertilidade específica (- - ), sobrevivência observada (•••) e sobrevivência estimada com modelo de distribuição de Weibull (-) para a população de Tetranychus urticae em jambu (Acmella oleracea). No canto superior direito, equação de distribuição de frequência de Weibull $(S)$, onde $t=$ tempo, coeficiente de regressão não linear $\left(R^{2}\right)$ e intervalo de confiança a $95 \%\left(\mathrm{IC}_{95 \%}\right)$ para o $\mathrm{R}^{2}$. Condições ambientais no laboratório: $\mathrm{T}$ $=27,8 \pm 2,78^{\circ} \mathrm{C}, \mathrm{UR}=76,7 \pm 16,21 \%$ e fotoperíodo de aproximadamente $12 \mathrm{~h}$. urticae em variedades de Rosa spp. e em cultivares de $S$. melongena.

Com base nos dados apresentados, T. urticae pode ocorrer em jambu, na região do estudo, ao longo de todo o ano. Porém, nos meses de menor umidade, que também coincidem com o pico do verão local, há um alto potencial para esse ácaro atingir o status de praga na cultura.

\section{Conclusões}

O jambu passa a fazer parte da lista de plantas hospedeiras de $T$. urticae. Esse ácaro pode causar danos a cultura, tendo um alto potencial para atingir o status de praga na região do estudo, sobretudo nos meses de temperatura elevada e menor umidade relativa do ar (verão).

$\mathrm{O}$ rápido desenvolvimento, a longa sobrevivência, a alta fecundidade, a taxa intrínseca de crescimento positiva, a taxa finita de aumento superior a um e o tipo da curva de sobrevivência, confirmam o potencial de crescimento populacional de T. urticae em jambu.
Valores altos para os coeficientes de regressão não linear indicam que a distribuição descreveu os dados de maneira satisfatória (Eliopoulos, Stathas \& Bouras, 2005). O parâmetro de escala é inversamente relacionado à taxa de mortalidade, dessa forma, quanto maior que um, mais lento é o declínio da população (Kontodimas et al., 2007). Assim, na curva de sobrevivência do tipo I, onde a taxa de mortalidade aumenta com o tempo, com a mortalidade ocorrendo principalmente em indivíduos idosos (Southwood \& Henderson, 2000). Portanto, a maioria das fêmeas morreram após os períodos de pré-oviposição e oviposição. Golizadeh et al. (2017) e Kumral et al. (2019) observaram sobrevivência semelhante para $T$.

\section{Agradecimentos}

A Técnica de Agropecuária do ICET-UFAM Luzia Corrêa Dunnemann pelos tratos culturais nos canteiros com jambu, utilizados para fornecer ramos e folhas para manutenção da criação de T. urticae e estudos biológicos.

\section{Literatura Citada}

ABD EL-WAHED, N. M.; EL-HALAWANY, A. S. 2012. Effect of temperature degrees on the biology and life table parameters of Tetranychus urticae Koch on two pear varieties. Egyptian Academic Journal of Biological Sciences 4(1):103-109. 
ABREU, F. A. et al. 2014. Influência da precipitação pluvial na abundância de ácaros em cafeeiro. Coffee Science 9(3):329-335.

ALI, A. H. et al. 2017. Temperature effects on some life table parameters of Tetranychus urticae Koch (Acari: Tetranychidae). Assiut Journal of Agricultural Sciences 48(1):163-172.

AUGER, P. et al. 2013. Evidence for synonymy between Tetranychus urticae and Tetranychus cinnabarinus (Acari, Prostigmata, Tetranychidae): review and new data. Acarologia 53(4):383-415.

BOUDREAUX, H. B. 1958. The effect of relative humidity on egg laying, hatching and survival in various spider mites. Journal of Insect Physiology 2(1):65-72.

BOURAS, S. L.; PAPADOULIS, G. T. 2005. Influence of selected fruit tree pollen on life history of Euseius stipulatus (Acari: Phytoseiidae). Experimental \& Applied Acarology 36(1-2):1-14.

CARDOSO, M. O.; GARCIA, L. C. 1997. Jambu (Spilanthes oleracea L.). In: Cardoso, M. O. (Coord.). Hortaliças não-convencionais da Amazônia. Brasília, Embrapa-SPI e Manaus, Embrapa-CPAA. pp.133-140.

EHI-EROMOSELE, C. O.; NWINYI, O. C.; AJANI, O. O. 2013. Integrated pest management. In: Soloneski, S.; Larramendy, M. (eds.). Weed and pest control: conventional and new challenges. Londres, IntechOpen. pp.105-115.

ELIOPOULOS, P. A.; STATHAS, G. J.; BOURAS, S. L. 2005. Effects and interactions of temperature, host deprivation and adult feeding on the longevity of the parasitoid Venturia canescens (Hymenoptera: Ichneumonidae). European Journal of Entomology 102(2):181-187.

FAVORETO, R.; GILBERT, B. 2010. Acmella oleracea (L.) R.K. Jansen (Asteraceae) - Jambu. Revista Fitos (Brasil) 5(1):83-91.

GOLIZADEH, A. et al. 2017. Comparative life table analysis of Tetranychus urticae Koch (Acari: Tetranychidae) on ten rose cultivars. Acarologia 57(3):607-616.

HAZAN, A.; GERSON, U.; TAHORI, A. S. 1973. Life history and life tables of the carmine spider mite. Acarologia 15(3):414-440.
HOMMA, A. K. O. et al. 2014. Etnocultivo do jambu para abastecimento da cidade de Belém, estado do Pará. In: Homma, A. K. O. (ed.). Extrativismo vegetal na Amazônia: história, ecologia, economia e domesticação. Brasília, DF, Embrapa. pp.329343.

HOY, M. A. 2011. Agricultural acarology: Introduction to integrated mite management. Boca Raton, CRC Press. 410p.

INSTITUTO NACIONAL DE METEOROLOGIA INMET. 2020. BDMEP - Banco de Dados Meteorológicos para Ensino e Pesquisa. Disponível em:http://www.inmet.gov.br/portal/ index.php?r=bdmep/bdmep. Acesso em: 03 fev. 2020.

JEPPSON, L. R.; KEIFER, H. H.; BAKER, E. W. 1975. Mites injurious to economic plants. University of California, Press Berkeley. 641p.

KAUR, P.; ZALOM, F. G. 2018. Development of twospotted spider mite, Tetranychus urticae Koch, at different temperatures. Agricultural Research Journal 55(1):172-174.

KIM, J. et al. 2008. Effect of temperature on development and life table parameters of Tetranychus urticae Koch (Acari: Tetranychidae) reared on eggplants. Korean Journal of Applied Entomology 47(2):163-168.

KLUBERTANZ, T. H.; PEDIGO, L. P.; CARLSON, R. E. 1990. Effects of plant moisture stress and rainfall on population dynamics of the two spotted spider mite (Acari: Tetranychidae). Environmental Entomology 19(6):1773-1779.

KONTODimAS, D. C. et a1. 2007. Life table parameters of the pseudococcid predators Nephus includens and Nephus bisignatus (Coleoptera: Coccinelidae). European Journal of Entomology 104(3):407-415.

KUMRAL, N. A. et al. 2019. Life table of Tetranychus urticae (Koch) (Acari: Tetranychidae) on different Turkish eggplant cultivars under controlled conditions. Acarologia 59(1):12-20.

LEWIS, W. H. et al. 1988. Mapas de distribuição geográfica de Acmella oleracea (L.) R. K. Jansen. Missouri Botanical Garden. Disponível em: http:// www. mobot1.mobot.org/website/map_post.asp. Acesso em: 18 out. 2019. 
MAIA, H. N. M.; LUIZ, A. J. B.; CAMPANHOLA, C. 2000. Statistical inference on associated fertility life table parameters using Jackknife technique: computational aspects. Journal of Economic Entomology 93(2):511-518.

MAULA, F.; KHAN, I. A. 2016. Effect of temperature variation on the developmental stages of Tetranychus urticae Koch and Panonychus ulmi Koch (Tetranychidae: Acarina) under laboratory conditions in Swat valley of Khyber Pakhtunkhwa, Pakistan. Journal of Entomology and Zoology Studies 4(1):279-283.

MIAO, W.; CHIOU, P. 2008. Confidence intervals for the difference between two means. Computational Statistics \& Data Analysis 52(4):2238-2248.

MIGEON, A. 2015. The Jean Gutierrez spider mite collection. Zookeys 489:15-24.

MIGEON, A.; DORKELD, F. 2019. Spider Mites Web: a comprehensive database for the Tetranychidae. Disponível em: http://www.montpellier.inra.fr/ CBGP/spmweb. Acesso em: 23 set. 2019.

OLKIN, I; FINN, J. D. 1995. Correlation redux. Psychological Bulletin 118(1):155-164.

MORAES, G. J.; FLECHTMANN C. H. W. 2008. Manual de acarologia: acarologia básica e ácaros de plantas cultivadas no Brasil. Ribeirão Preto, Holos. 308p.

PAL, P. K. et al. 1989. Approaches to integrated control of Tetranychus cinnabarinus on eggplant. In: Channabasavanna, G. P.; Viraktamath, C. A. (ed.). Progress in Acarology. New Delhi, Oxford and IBH Publishing. pp.455-459.

PINDER, J. E.; WIENER, J. G.; SMITH, M. H. 1978. The Weibull distribution: a new method of summarizing survivorship data. Ecology 59(1):175-179.

PRITCHARD, A. E.; BAKER, E. W. 1955. A revision of the spider mite family Tetranychidae. San Francisco, Pacific Coast Entomological Society (Memoirs series, 2). 472p.

R CORE TEAM. 2018. R: A language and environment for statistical computing. R Foundation for Statistical Computing, Vienna, Austria. URL https://www.Rproject.org/.
RÉAUMUR, R.A. 1735. Observations du Thermometre, faites à Paris pendant l'année M.DCCLXXXV comparées avec celles qui ont été faites sous la Ligne; à l'Isle de France, à Alger et en quelquesunes de nos Isles de l'Amérique. Memoires de l'Académie Royale des Sciences. pp. 545-576.

REIS, P. R.; HADDAD, M. L. 1997. Distribuição de Weibull como modelo de sobrevivência de Iphiseiodes zuluagai Denmark \& Muma (Acari: Phytoseiidae). Anais da Sociedade Entomológica do Brasil 26(3):441-444.

RIAHI, E. et al. 2013. Temperature effects on development and life table parameters of Tetranychus urticae (Acari: Tetranychidae). Journal of Agricultural Science and Technology 15(4):661-672.

SCOTT, A. J.; KNOTT, M. 1974. A cluster analysis method for grouping means in the analysis of variance. Biometrics 30(3):507-512.

SILVA, E. A. et al. 2009. Tetranychus urticae (Acari: Tetranychidae) on Gerbera jamesonii Bolus and Hook (Asteraceae). Brazilian Journal of Biology 69(4):1121-1125.

SOUTHWOOD, T. R. E.; HENDERSON, P. A. 2000. Ecological methods. 3 ed. Oxford, Blackwell. 575p.

STEIN, D. 2006. Five steps of IPM help reduce pesticide use. Journal of Pesticide Reform 26(3):11-11.

TAN, S. H.; TAN, S. B. 2010. The correct interpretation of confidence intervals. Proceedings of Singapore Healthcare 19(3):276-278.

UDDIN, M. N. et al. 2015. Life table parameters of Tetranychus urticae Koch (Acari: Tetranychidae) on different bean varieties. African Entomology 23(2):418-426.

VASCONCELOS, G. J. N.; LIMA, S. C.; SILVA, N. M. 2012. Ocorrência do ácaro branco Polyphagotarsonemus latus (Banks) em jambu Spilanthes oleracea e outras plantas hospedeiras no estado do Amazonas. Revista Agroecossistemas (Brasil) 4(1):81-86.

ZUCCHI, R. A. 1990. A taxonomia e o manejo de pragas. In: Crocomo, W. B. (org.). Manejo Integrado de Pragas. UNESP, Botucatu, São Paulo, CETESB. pp.57-70. 